\title{
Interactive comment on "Characteristics and sources of aerosol aminiums over the eastern coast of China: Insights from the integrated observations in a coastal city, adjacent island and the marginal seas" by Shengqian Zhou et al.
}

Anonymous Referee \#2

Received and published: 16 May 2019

This manuscript reports observations of particle phase aminium ions from two ground sites (Shanghai and Huaniao Island) providing a full annual cycle, and one ship platform during a springtime cruise in the Yellow and East China Seas. Given that amines are thought to be important in new particle formation, and that there are still relatively few reports of their concentrations in the gas and particle phases, these data are a useful contribution.

The description of the chemical analysis for amines is missing some important informaDiscussion paper tion: 1) What is the full suite of aminium ions that could be detected (and was calibrated 
for) using the analytical method?

2) What were the limits of detection for the measurements of each aminium ion that was measured?

3) How were measurements that we below the detection limit incorporated into the subsequent data analysis, including the calculations of the mean and standard deviations at each site in each season.

This information is especially important because the authors go on to compare their observations with those reported from other studies. If their analysis technique was capable of measuring monomethyl- and monoethyl-amine (Lines 168-170), but did not find them above the detection limit, this is important information to include. The detection limits for the species should be included explicitly in the manuscript.

In Section 3.2, the authors correlate the speciated aminium loadings in the particle phase with various environmental variables. Given the time-integrated nature of the particle collection, some discussion should be made to the impact of averaging the variables over a full 24- or 48-hour collection interval.

I do not find the analysis on the impact of oxidation on the aminium ions presented in Section 3.2.3 and Figure 3c and Figure 4 to be convincing. The relationship between the ratio of aminium/ $\mathrm{NH} 4$ versus ozone is only significant for $\mathrm{TEAH}+$. For the other two aminiums, the need to separately derive a slope for a subset of the high ozone data suggests that the analysis is not robust. The accompanying text is too speculative. Similarly, the anti-correlation between particle phase aminiums and formate measured over the Yellow and East China Seas (Figure 4) could result from many different factors and there is no compelling evidence provided that it results from photo-oxidation.

In Section 3.4.3 the authors present an interesting approach to deriving the relative marine versus terrestrial contributions to the particle phase aminium ions by examining the relationship between the ratio of aminium/ $\mathrm{NH} 4+$ to MSA/SO42-. The strong 
relationship between these two ratios indicates the possible value of this approach. However I wonder if the authors have considered the following factors in extracting quantitative values from this method: 1) while MSA and sulfate both have very low volatility, ammonia and amines are very volatile, therefore the particle phase measurements of the S-containing species are likely very consistent with the emission ratios (of DMS and SO2), whereas the measured ratio of particle phase aminium/ $\mathrm{NH} 4+$ may not correspond very closely to the emission ratios of amines and ammonia; this is in part because 2) the thermodynamic favourability of gas-particle partitioning the amines and ammonia are slightly different (depending on the phase and $\mathrm{pH}$ of particles), so the observed aminium/NH4+ ratio could vary with the chemistry of the particles and not just the emission ratios of amines and ammonia. Can the authors comment on how much this might influence the robustness of their terrestrial vs marine source apportionment?

Specific comments:

Section 3.2.1 - 'Diffusion' is not the right term to distinguish the differences in dilution or ventilation under different wind speed and boundary layer height conditions.

Figure 3 caption should specify that this analysis is only for the Shanghai data.

Figure 7 - would be easier to read if there was a line (or a different symbol) indicating the average value for a rather than a bar that arbitrarily extends to a value of 1 .

Line 292 - 'fold' should be 'factor'

Line 296 - 'folds' should be 'times'

Interactive comment on Atmos. Chem. Phys. Discuss., https://doi.org/10.5194/acp-2019-107, 2019. 